\title{
Commentaries
}

\section{The role of genetic diversity in cancer}

\author{
Lauren M.F. Merlo' and Carlo C. Maley',2
}

\author{
${ }^{1}$ Molecular and Cellular Oncogenesis Program, The Wistar Institute, Philadelphia, Pennsylvania, USA. ${ }^{2}$ Genomic and Computational Biology \\ Graduate Program and Cell and Molecular Biology Graduate Program, University of Pennsylvania, Philadelphia, Pennsylvania, USA.
}

\begin{abstract}
The role of genetic heterogeneity within neoplasms is increasingly recognized as important for understanding the dynamics of cancer progression, cancer stem cells, and therapeutic resistance, and there is interest in intratumoral heterogeneity measurements as potential biomarkers for risk stratification. In this issue of the JCI, Park et al. characterize this genetic diversity in carcinoma in situ and in invasive regions from 3 types of human breast cancers and lay the groundwork for translation of these measures to the clinic.
\end{abstract}

Although there are clear suggestions that diversity may be important in the establishment and progression of cancers (1), it is unclear how diversity should be defined. Should we use genetic or phenotypic markers? How can we account for epigenetic diversity? Should we look at large samples or small ones, and how many biopsies should we sample in order to accurately estimate the underlying diversity of the neoplasm? The problems of defining diversity and characterizing the number of cancer clones are not new and have parallels to problems encountered in other fields. Ecologists have created various measures to estimate the number and abundance of species (2); even in literature, calculating the number of words Shakespeare knew from his existing corpus (3) is analogous to determining the number of cancer clones in a tumor from a small number of samples. Heterogeneity in neoplasms has long been recognized (4-7), but has only recently been systematically investigated, and much can be gained by applying these methodologies to cancer and precancerous lesions $(8-10)$. As Park et al. clearly demonstrate in their study in this issue of the JCI (11), this is not merely an academic exercise, as the level of heterogeneity in a neoplasm may have important clinical implications as a biomarker of risk stratification.

\section{Genetic diversity as a biomarker}

It is important to note that measures of genetic or epigenetic diversity are a novel form of biomarker. Most previous biomarkers in cancer measure the presence of an abnormality, like loss of heterozygosity

Conflict of interest: The authors have declared that no conflict of interest exists.

Citation for this article: J Clin Invest. 2010; 120(2):401-403. doi:10.1172/JCI42088.
(LOH) in the tumor suppressor TP53 (12), amplification of the tyrosine kinase receptor HER2, or the level of some mRNA or protein, such as prostate-specific antigen (13). In contrast, by measuring genetic diversity, we are measuring a parameter of the underlying dynamics driving neoplastic progression, regardless of the particular loci in question. Because neoplastic progression is a process of somatic evolution, in which genetic and epigenetic alterations generate diversity, and ecological interactions like competition between clones lead to natural selection among that diversity, the generation and maintenance of diversity in heritable alterations, such as genetic and epigenetic changes, determines the rate of somatic evolution. Thus, it is possible to gather information on the rate of progression even if we do not know the specific tumor suppressor genes and oncogenes (i.e., driver mutations) that provide fitness benefits to neoplastic clones in a given organ. Other measures of the rate of somatic evolution, such as mutation rate, population size of self-renewing cells, and generation time, should also be robust and general biomarkers of progression (14).

We have previously shown that genetic clonal diversity is predictive of progression in a precancerous lesion (10). In their current study, Park et al. extended this idea to cancers, characterizing heterogeneity from 4 samples from each of 15 human breast cancer lesions, including a mix of ductal carcinoma in situ (DCIS; the most common type of noninvasive breast cancer) and invasive regions (11). They report a high level of heterogeneity within the tumor samples, and their analyses hint that measurements of the diversity in one phenotypic subpopulation (CD24+ cells in DCIS) may be a useful predictor of a variety of histopathological characteristics, such as tumor stage, presence of intratumoral and/or peritumoral DCIS, and estrogen receptor (ER) status. ER status is important because $\mathrm{ER}^{+}$tumors tend to be responsive to selective ER modulators, such as tamoxifen and raloxifene. Thus, it is possible that a single diversity measure could provide a snapshot of a variety of tumor processes. Surprisingly, they also found that diversity in DCIS and invasive regions is similar, which suggests that diversity does not increase in a systematic manner as the neoplasm becomes invasive. These studies together suggest that measurements of diversity may be clinically important at all phases of disease progression. The studies have used different types of lesions to measure diversity: microsatellite shifts, $\mathrm{LOH}$, mutation, methylation, and copy number variation measured by FISH (as in our previous work; see ref. 10). The fact that clinically relevant information can be extracted from diversity measures based on such a variety of markers implies that diversity measurements are potentially robust biomarkers.

\section{Cancer stem cells or independent clones?}

The present study by Park et al. extends previous work of the Polyak lab (15) questioning the cancer stem cell paradigm in breast cancers, an idea that has important implications for the evolutionary dynamics in neoplastic progression. The cancer stem cell hypothesis posits that only a minority of cells in a neoplasm are self-renewing and are responsible for the long-term propagation of the neoplasm, analogous to stem cells in normal tissues. In breast cancer, CD44 has been proposed as a marker of breast stem cells, while $\mathrm{CD} 24^{+}$cells are thought to derive from the CD44 $4^{+}$cell population. In their study, Polyak et al. found a clone in the $\mathrm{CD} 24^{+}$population not present in the $\mathrm{CD} 44^{+}$population (15), which suggests that the cancer stem cell and linear differentiation model is inaccurate in this case. If CD $24^{+}$cells have only diverged from $\mathrm{CD} 44^{+}$cells for a few cell divisions, then the $\mathrm{CD}_{4} 4^{+}$and $\mathrm{CD} 24^{+}$cells should have essentially the same genetic diversity (Figure 1). In the current study, Park et al. found that 


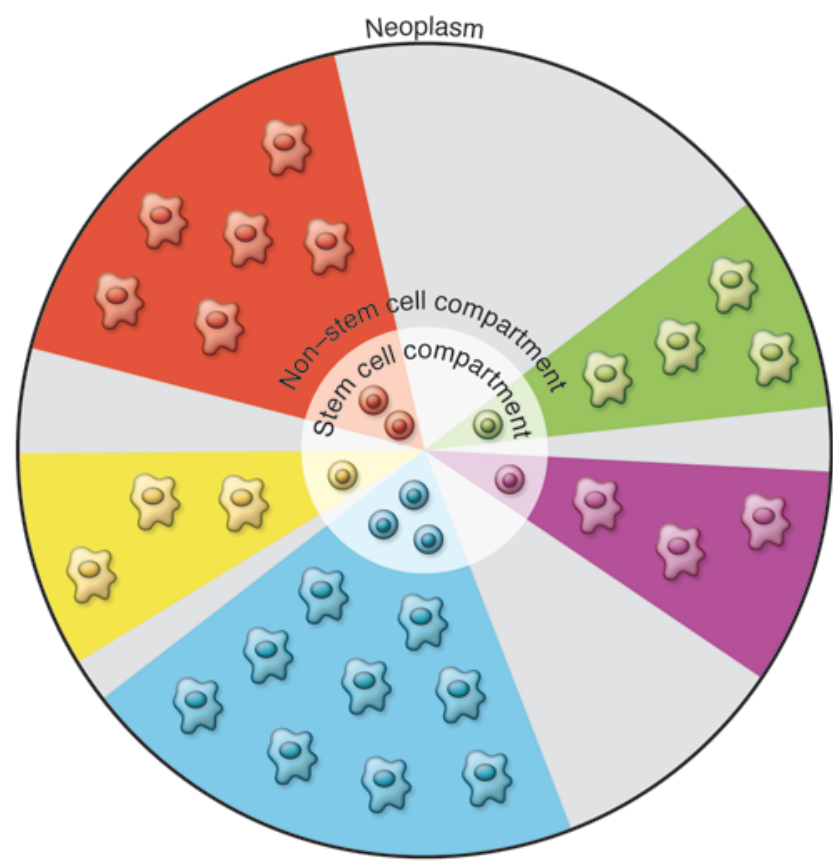

Figure 1

For neoplasms maintained by stem cells, somatic evolution will be dominated by evolution within the stem cell compartment. Here, the blue stem cell subclone may have a competitive advantage and expand relative to the other stem cell subclones. Thus, the cancer stem cell hypothesis and the somatic evolutionary theory of cancer are not mutually exclusive. Because the non-stem cells are, by definition, not self-renewing, they are evolutionary dead ends. However, the genetic diversity in the non-stem cell compartment should reflect the genetic diversity in the stem cell compartment, with the exception of differences in the proliferation rates of the different non-stem cell subclones, which might slightly skew their frequencies relative to the stem cell pool, and rare genetic alterations that occur during the few cell divisions that separate a non-stem cell from its stem cell ancestor. If neoplastic non-stem cells can revert to a stem cell phenotype, then the evolutionary dynamics become more complex and some of the non-stem cells may not be evolutionary dead ends. The results reported by Park et al. in this issue of the $\mathrm{JCl}$ (11) suggest that CD44 and CD24 do not distinguish between stem cells and non-stem cells in breast cancer.

diversity generally differs, sometimes dramatically, between $\mathrm{CD} 24^{+}$and $\mathrm{CD} 44^{+}$cells (see Figure 5A in ref. 11). Either the linear differentiation model needs to be reevaluated, or we need to develop better markers of breast cancer stem cells. The authors also found diversity differences between cells in invasive and DCIS areas, although it can be difficult to interpret this in relation to the cancer stem cell hypothesis. If the stem cell pool is large enough (16) or the mutation rate high enough, a neoplasm might contain a highly diverse pool of cells.

The cancer stem cell hypothesis and the theory of clonal evolution are not mutually exclusive (Figure 1). The presence of cancer stem cells simply moves the dynamics of evolution from the cancer cell population as a whole to the stem cell compartment. Thus, clonal expansion and competition would occur within the stem cell pool. This may make genetic drift (i.e., stochastic changes in mutation frequency) a more important process in neoplastic progression when the total pool of evolving cells is small. The dynamics of putative cancer stem cell compartments are largely unknown. If a stem cell pool is to expand, stem cells must divide symmetrically at least some of the time and either replace slower-dividing cells or expand the population size of the stem cell compartment. We do not yet know whether the putative stem cell pool in a neoplasm acts as a single population or if there exists a variety of potentially cooperative or competitive subpools of stem cells. Stem cell-like behavior may also be a product of the microenvironment, and the feedback between microenvironment and cancer initiation, invasion, and metastasis is an area of active study $(17,18)$.

\section{Open questions}

To our knowledge, measures of heterogeneity have not been systematically studied in cancer itself until now. Several key areas remain unexplored. Most importantly, the relationship between diversity measurements in cancer samples and survival has yet to be systematically investigated. Our previous study demonstrated a link between progression and diversity in precancerous esophageal neoplasms (10), but it is difficult to measure most tumors in their precancerous states. With only a small number of samples, the data reported by Park et al. hints at a link between diversity and histopathology (11), but this needs to be evaluated in a larger sample pool and correlated with longitudinal follow-up, including treatment outcome. A second key area yet to be examined is the relationship between genetic diversity and therapeutic resistance. In studying resistance, we are battling the underlying evolutionary forces that lead to diversity in a cancer, the interplay between mutation and selection. It is clear that therapy can select for the expansion of rare clones (19, $20)$. Logic would suggest that more heterogeneous cancers are more likely to harbor one of these resistance mutations, but this has yet to be demonstrated. Finally, determining the best measures of heterogeneity and loci must take into account their robustness as biomarkers and ease of clinical translation.

The study presented here (11) provides a glimpse of the high level of genetic heterogeneity found in stem cell-like and more differentiated populations from invasive and in situ breast cancer lesions. This should be the first of many such studies to elucidate the role and dynamics of diversity within neoplasms.

\section{Acknowledgments}

This work was supported in part by the Commonwealth Universal Research Enhancement Program, Pennsylvania Department of Health; the Pew Charitable Trust; the Martha W. Rodgers Charitable Trust; the Landon AACR Innovator Award for Cancer Prevention; and NIH grants R03 CA137811, P01 CA91955, P30 CA010815, F32 CA132450, and R01 CA140657.

Address correspondence to: Carlo C. Maley, The Wistar Institute, 3601 Spruce St., Philadelphia, Pennsylvania 19104, USA. Phone: (215) 495-6838; Fax: (215) 495-6829; E-mail: cmaley@alum.mit.edu. 
1. Heng HH, et al. Stochastic cancer progression driven by non-clonal chromosome aberrations. $J$ Cell Physiol. 2006;208(2):461-472.

2. Magurran AE. Measuring Biological Diversity. Malden, MA: Blackwell Publishing; 2004.

3. Efron B, Thisted R. Estimating the number of unseen species: How many words did Shakespeare know? Biometrika. 1976;63:435-447.

4. de Grouchy J, de Nava C, Cantu JM, Bilski-Pasquier G, Bousser J. Models for clonal evolutions: a study of chronic myelogenous leukemia. Am J Hum Genet. 1966;18(5):485-503

5. Ford CE, Clarke CM. Cytogenetic evidence of clonal proliferation in primary reticular neoplasms. Can Cancer Conf. 1963;5:129-146.

6. Dawe CJ. Phylogeny and oncogeny. Natl Cancer Inst Monogr. 1969;31:1-40.

7. Yosida TH. Relation between chromosomal alteration and development of tumors. Jpn J Genet. 1966:41:439-451.

8. Siegmund KD, Marjoram P, Woo YJ, Tavare S, Shibata D. Inferring clonal expansion and cancer stem cell dynamics from DNA methylation patterns in colorectal cancers. Proc Natl Acad Sci US A. 2009;106(12):4828-4833.

9. Tsao JL, Tavare S, Salovaara R, Jass JR, Aaltonen LA, Shibata D. Colorectal adenoma and cancer divergence. Evidence of multilineage progression. Am J Pathol. 1999;154(6):1815-1824.

10. Maley CC, et al. Genetic clonal diversity predicts progression to esophageal adenocarcinoma. Nat Genet. 2006;38(4):468-473.

11. Park SY, Gönen M, Kim HJ, Michor F, Polyak K. Cellular and genetic diversity in the progression of in situ human breast carcinomas to an invasive phenotype. J Clin Invest. 2010;120(2):636-644.

12. Galipeau PC, et al. NSAIDs modulate CDKN2A, TP53, and DNA content risk for progression to esophageal adenocarcinoma. PLoS Med. 2007;4:e67.

13. Ludwig JA, Weinstein JN. Biomarkers in cancer staging, prognosis and treatment selection. Nat Rev Cancer. 2005;5(11):845-856

14. Pepper J, Findlay CS, Kassen R, Spencer S, Maley C. Cancer research meets evolutionary biology. Evolu- tionary Applications. 2009;2(1):62-70. doi:10.1111/ j.1752-4571.2008.00063.

15. Shipitsin M, et al. Molecular definition of breast tumor heterogeneity. Cancer Cell. 2007;11(3):259-273.

16. Quintana E, Shackleton M, Sabel MS, Fullen DR, Johnson TM, Morrison SJ. Efficient tumour formation by single human melanoma cells. Nature. 2008; 456(7222):593-598

17. Hu M, Polyak K. Microenvironmental regulation of cancer development. Curr Opin Genet Dev. 2008;18(1):27-34.

18. Polyak K, Weinberg RA. Transitions between epithelial and mesenchymal states: acquisition of malignant and stem cell traits. Nat Rev Cancer. 2009; 9(4):265-273.

19. Roche-Lestienne C, Preudhomme C. Mutations in the ABL kinase domain pre-exist the onset of imatinib treatment. Semin Hematol. 2003;40(2 Suppl 2):80-82.

20. Shah NP, et al. Sequential ABL kinase inhibitor therapy selects for compound drug-resistant BCRABL mutations with altered oncogenic potency. J Clin Invest. 2007;117(9):2562-2569.

\title{
TGF- $\beta$ in the pathogenesis and prevention of disease: a matter of aneurysmic proportions
}

\author{
Harry C. Dietz
}

Institute of Genetic Medicine and Howard Hughes Medical Institute, Johns Hopkins University School of Medicine, Baltimore, Maryland, USA.

TGF- $\beta$ regulates many aspects of cellular performance relevant to tissue morphogenesis and homeostasis. Postnatal perturbation of TGF- $\beta$ signaling contributes to the pathogenesis of many disease states, as recently exemplified through the study of Marfan syndrome (MFS), including aortic aneurysm and skeletal muscle myopathy. Heterogeneity in the regulation and consequences of TGF- $\beta$ signaling, amplified in the context of disease, has engendered confusion and controversy regarding its utility as a therapeutic target. Three studies recently published in the JCI, including one in this issue, underscore the complexity of this subject. Heydemann and colleagues implicate dimorphic variation in latent TGF- $\beta$-binding protein 4 (LTBP4), a regulator of TGF- $\beta$ bioavailability and activation, as a modifier of muscular dystrophy in $\gamma$-sarcoglycan-deficient mice. In contrast to experience with ascending aortic aneurysm in MFS, Wang and colleagues show that systemic abrogation of TGF- $\beta$ signaling worsens (rather than attenuates) Ang II-induced abdominal aortic aneurysm progression in mice. Tieu and colleagues define alterations in the regulation of vascular inflammation in the pathogenesis of Ang II-induced aneurysm and dissection in mice, which may help shed some light on this apparent paradox.

Historically, perturbation (largely enhancement) of TGF- $\beta$ signaling has been strongly implicated in the pathogenesis of diverse disease states, prominently including the initiation and progression of cancer and tissue fibrosis. Despite decades of intensive study in these contexts, the net effects

Conflict of interest: The author has declared that no conflict of interest exists.

Citation for this article: JClin Invest. 2010; 120(2):403-407. doi:10.1172/JCI42014 of TGF- $\beta$ signaling in disease pathogenesis and, perhaps more importantly, the incorporation of its antagonism into therapeutic strategies, remain controversial. This is nicely illustrated by the so-called TGF- $\beta$ cancer paradox. In brief, TGF- $\beta$ plays a prominent role in the suppression of tumorigenesis through induction of cellcycle arrest and apoptosis and maintenance of cellular differentiation, as evidenced by the frequent biallelic loss of genes encoding TGF- $\beta$ receptors or intracellular media- tors of signaling in multiple tumor types (for example, see ref. 1). Attenuation or loss of tumor responsiveness can induce upregulation of TGF- $\beta$ ligand expression, resulting in excessive stimulation of the signaling-intact neighboring stroma (reviewed in ref. 2). Attributable consequences include impairment of tumor surveillance through inhibition of adaptive immunity, acceleration of tumor growth through enhancement of angiogenesis, and induction of tumor invasion and metastasis through stimulation of innate immunity (including mast cell, monocyte, and macrophage chemotaxis), compromising of endothelial boundaries, and promotion of epithelial- or endothelialmesenchymal transition (EpMT [ref. 2] and EnMT [ref. 3], respectively; collectively EMT). Amplification of TGF- $\beta$ signaling can occur due to enhanced TGF- $\beta$ ligand expression (e.g., by recruited mast cells or macrophages) or activation (e.g., by MMPs or simple fibrosis-dependent enhancement of shear stress within the tumor microenvironment; ref. 4). Additional TGF- $\beta$-related paradoxes are evident. For example, TGF- $\beta$ can either induce or suppress angiogenesis depending upon its concentration and repertoire of TGF- $\beta$ receptors and accessory proteins (2). 